\title{
Backhousia citriodora Leaf Oil
}

National Cancer Institute

\section{Source}

National Cancer Institute. Backhousia citriodora Leaf Oil. NCI Thesaurus. Code C107268.

The essential oil of Backhousia citriodora. One form of lemonmyrtle oil is used as a flavoring or for its aromatic properties. The other form of lemon myrtle oil is used in the production of citronella. 